\title{
Interaction of Paraffin Wax Gels with Ethylene/Vinyl Acetate Co-polymers
}

\author{
Henry S. Ashbaugh, ${ }^{\dagger, \dagger}$ Xuhong Guo, Dietmar Schwahn,,$^{\dagger}$ \\ Robert K. Prud'homme, ${ }^{* \dagger}$ Dieter Richter, ${ }^{\S}$ and Lewis J. Fetters ${ }^{\#}$ \\ Department of Chemical Engineering, Princeton University, Princeton, New Jersey 08544, \\ Institute for Solid State Research, Research Center, Jülich GmbH, D-52425, Jülich, Germany, \\ and School of Chemical and Biomolecular Engineering, Cornell University, \\ Ithaca, New York 14853-5201
}

Received April 13, 2004. Revised Manuscript Received September 15, 2004

\begin{abstract}
The commercial grades of ethylene/vinyl acetate (EVA) co-polymers have found application as "pour point" depressants in refined fuels. This study focuses on their behavior as additives to crude oils, where the intent is to reduce the yield stress of the gels that can form when the oil exits the reservoir. The model crude oils consisted of $4 \mathrm{wt} \%$ wax in decane. At EVA dosage levels of $\sim 200 \mathrm{ppm}$, the reduction in yield stress is 3 orders of magnitude for the $\mathrm{C}_{36}$ wax, whereas the reduction is 1 order of magnitude for $\mathrm{C}_{32}$ and only 3 -fold for the $\mathrm{C}_{28}$ wax. This decrease in efficiency with decreasing wax carbon number indicates that the EVA materials would not provide an adequate reduction in yield stress to ensure against gelation in pipeline transport. Neutron scattering studies, as a function of temperature, of the self-assembly of the EVA co-polymers show dramatically different aggregated structures in decane. The EVA with the lowest ethylene content shows scattering that increases with a power-law exponent of $\sim 1.6$. This scattering behavior is typical for weakly aggregating polymer gels. In contrast, the EVA with the higher ethylene content shows a transition from surface scattering (found for strongly segregated objects) to a plateau whose height is dependent on temperature. Micrographs of the wax crystal morphology indicate that the ethylene-poor EVA alters the wax crystal habit at higher concentrations more effectively than does its higher-ethylene-content counterpart, whereas the latter EVA grade seems to form more wax crystals at low concentrations.
\end{abstract}

\section{Introduction}

The crystallization of waxes from solutions is of practical interest and relevance in the petroleum industry. The systems in question include the upstream paraffinic crude oils and downstream refined products (middle distillates), such as diesel fuel and kerosene, along with lube and heating oils. Unmodified, these systems, over the temperature range of $\sim 0-25{ }^{\circ} \mathrm{C}$, will either gel or produce insoluble wax crystals that, in turn, will plug pumps, transmission lines, and filters. The paraffin waxes show a variety of crystallization forms from the melt or from solution, with the most frequent being the orthorhombic, hexagonal, monoclinic, and triclinic morphologies.

The ethylene/vinyl acetate (EVA, Infinium, Ltd.) copolymers are chains with polyethylene sections of varying length, depending on the amount of co-polymerized vinyl acetate (VA) co-monomer. The VA units in the commercial products are nominally present at $\sim 9 \%$ (mole fraction) in the EVA-nu, $\sim 12 \%$ in the EVA-ss, and $\sim 15 \%$ in the EVA-ga. (The acronyms nu, ss, and ga

* Author to whom correspondence should be addressed. E-mail: prudhomm@princeton.edu.

$\dagger$ Princeton University.

* Present address: Department of Chemical and Biomolecular

Engineering, Tulane University, New Orleans, LA 70118.

\& Institute for Solid State Research.

\# Cornell University. denote the nucleator, single-shot, and growth-arrestor grades. Each grade denotes the perceived function of each co-polymer type.) This family of materials has been a long-term mainstay additive in middle distillate fuels ${ }^{1,2}$ and has had varying degrees of success in controlling the size of the wax crystals formed in situ. When successful as an additive, EVA produces crystals that are much smaller and more numerous than those found in the unmodified system. The crystals also exhibit a more compact habit. ${ }^{3}$ The commercial packages usually consist of EVA-ss or mixtures of the nu and ga grades. The ss grade is assumed to combine both the nucleator and growth arrestor characteristics found in the EVA mixture. A complementary structure is the semicrystalline:amorphous diblock $(\sim 1.5 \mathrm{~K}-5 \mathrm{~K})$ of polyethylene-(ethylene-propylene) (PE-PEP) ${ }^{4}$ where the PEP segment was derived from 1,4-polyisoprene. This type of polyolefin diblock has been observed to exhibit success as both an upstream and downstream additive for wax crystal control. ${ }^{5,6}$

(1) Owen, K. Gasoline and Diesel Fuel Additives; Wiley, New York, 1989

(2) Lewtas, K.; Tack, R. D.; Beiny, D. H. M.; Mullin, J. W. In Advances in Industrial Crystallization; Garside, J., Davey, R. J., Jones, A. G., Eds.; Butterworth-Heinemann: London, 1991.

(3) Petinelli, J. C. Rev. Inst. Fr. Petr. 1979, 34, 791

(4) Richter, D.; Schneiders, D.; Monkenbusch, M.; Willner, L.; Fetters, L. J.; Huang, J. S.; Lin, M.; Moretenson, K.; Farago, B. Macromolecules 1997, 30, 1053. 
The molecular parameters of the EVA co-polymer, the composition of the waxes, and the identity of the solvent phase-the oil or the fuel-have roles in the efficiency (or lack thereof) of the co-polymer additive. The EVA materials are derived from a high-pressure:high-temperature co-polymerization, where the resulting length of the methylene segments is dependent on the relative concentrations of the two monomers. Because ethylene and vinyl acetate have similar reactivity ratios, ${ }^{7}$ the vinyl acetate distribution, in principle, should be essentially random and the mean methylene segment length controlled by the VA content of the co-polymer.

This work examines the EVA-ga and the EVA-ss copolymers, with regard to their influence on the yield stress of model crude oils that are composed of paraffin wax dissolved in decane, which readily form solidlike gels below the wax precipitation temperature. Those results are, in turn, compared to those found for the $\mathrm{PE}-\mathrm{PEP}$ diblocks ${ }^{6}$ and poly(ethylene butylene) (PEB$n$ ) random co-polymers ${ }^{8-10}$ (here, $n$ denotes the number of ethyl goups per 100 backbone carbons).

In this study, we consider gel formation from quiescent conditions, such as those that occur in a diesel motor or a subsea pipeline during shutdown. The effect of shear on the structure of wax gels has not been considered here but has been studied for oils without wax crystal modifiers by Singh, Fogler, and co-workers. ${ }^{11,12}$ Furthermore, this study addresses only a single cooling rate; we have addressed the role of cooling rate separately. ${ }^{13}$ For simplicity, we have also considered only single-component waxes. In actual crude oils, the distribution of wax components and polar asphaltenes create additional complexities and opportunities for further research. ${ }^{14,15}$ We have begun to address the role of wax mixtures and optimum polymer additives. ${ }^{16,17}$

\section{Experimental Section}

Samples. EVA random co-polymers were obtained from Infiniem, Ltd., and used as received. Waxy oil samples for measurement of the yield stress were prepared as mixtures of 4 wt \% straight-chain paraffin waxes in decane (SigmaAldrich) with variable concentrations of EVA-ss or EVA-ga. The EVA-nu grade was not used in these studies, because $\sim 50 \%$ of the material precipitated from solution. ${ }^{8}$ The straightchain paraffin waxes of carbon numbers $\mathrm{C}_{n}$ (where $n=28$, 32 , and 36) were examined (Sigma-Aldrich).

(5) Leube, W.; Monkenbusch, M.; Schneiders, D.; Richter, D.; Adamson, D.; Fetters, L. J.; Dounis, P.; Lovegrove, R. Energy Fuels 2000, 14, 419 .

(6) Ashbaugh, H. S.; Fetters, L. J.; Adamson, D. H.; Prud'homme, R. K. J. Rheol. 2002, 46, 763.

(7) Greenley, R. Z. Polymer Handbook, 4th ed.; Brandup, J., Immergut, E. H., Grulke, E. A., Eds.; Wiley: New York, 1999.

(8) Ashbaugh, H. S.; Radulescu, A.; Prud'homme, R. K.; Schwahn, D.; Richter, D.; Fetters, L. J. Macromolecules 2002, 35, 7044.

(9) Radulescu, A.; Schwan, D.; Monkenbusch, M.; Fetters, L. J.; Richter, D. J. Polym. Sci., Polym. Phys. Ed. 2004, 42, 3113.

(10) Schwahn, D.; Richter, D.; Wright, P. J.; Symon, C.; Fetters, L. J.; Lin, M. Macromolecules 2002, 35, 861.

(11) Singh, P.; Venkatesan, R.; Fogler, H. S.; Nagarajan, N. R. AIChE J. 2001, 47, 6.

(12) Singh, P.; Fogler, H. S.; Nagarajan, N. J. Rheol. 1999, 43, 1437.

(13) Guo, X.; Pethica, B. A.; Huang, J. S.; Adamson, D. H.; Prud'homme, R. K. Macromolecules, in press.

(14) Garcia, M. C. Energy Fuels 2000, 14, 1043.

(15) Venkatesan, R.; Oestlund, J.-A.; Chawla, H.;Wattana, P.; Nyden, M.; Fogler, S. Energy Fuels 2003, 17, 1630-1640.

(16) Guo, X.; Pethica, B. A.; Huang, J. S.; Prud'homme, R. K. Macromolecules 2004, 37, 5638.

(17) Guo, X.; Pethica, B. A.; Huang, J. S.; Prud'homme, R. K.; Adamson, D. H.; Fetters, L. J. Energy Fuels 2004, 18, 930.
Yield Stress Measurements. The yielding behavior of waxy oils is complex and akin to the deformation and fracture of ductile solids. ${ }^{18}$ Although compositionally homogeneous, compared to crude oil, our waxy decane solutions yield in a similar manner. In particular, after the wax gel network structure is broken by the application of stress, the wax particles settle and readily flow either with diminished or negligible yield stress. Here, we are concerned with the initial breakdown of the gel.

Yield stress measurements were performed for sample mixtures of 4 wt $\%$ paraffin wax in decane, as a function of EVA concentration at $0{ }^{\circ} \mathrm{C}$. Although the yield stress $\left(\tau_{y}\right)$ is defined as the stress below which no flow occurs, we adopt an operational definition of $\tau_{y}$ as the stress at the transition between the creep and liquidlike viscosity regimes. In this way, $\tau_{y}$ is identified as the stress for which the derivative $-\mathrm{d}(\ln \eta) /$ $\mathrm{d}(\ln \tau)$ is a maximum (where $\eta$ is the measured viscosity). These measurements are discussed in greater detail in ref 6 .

The yield-stress measurements were performed on a Rheometrics DSR controlled stress rheometer with parallel plate geometry. The temperature was controlled to within $0.1^{\circ} \mathrm{C}$ by a Peltier plate. To minimize evaporation, the sample was covered with a solvent trap. The samples were initially heated to $70^{\circ} \mathrm{C}$, to erase their thermal history and solubilize the EVA chains. After the samples were heated, the temperature was immediately decreased to $0{ }^{\circ} \mathrm{C}$, to precipitate the wax and form a solidlike gel. After the sample was allowed to anneal at constant temperature under no stress for $20 \mathrm{~min}$, a static stress was applied and incrementally increased every $10 \mathrm{~s}(100$ stress increments per decade) and the viscosity was measured. Typically, the initial applied stress was chosen well below the stress at which creep begins. Although there is concern over whether $\tau_{y}$ for crude oils is independent of the test geometry, ${ }^{18}$ we are primarily concerned with the order of magnitude of $\tau_{y}$ and comparison of varying sample compositions in the same geometry. The measured yield stresses were reproducible to within $10 \%-20 \%$.

Optical Microscopy. Wax crystal morphologies were observed using a Nikon model TE200 inverted microscope outfitted with phase and differential interference contrast (DIC) optics (Micron Optics, Cedar Knoll, NJ). Images were captured using a Kodak model ES 310 charge-coupled device (CCD) camera that was connected to a personal computer (PC) via a PIXCID imaging board (EPIX, Buffalo Grove, IL). Samples were initially heated to $70{ }^{\circ} \mathrm{C}$ and then quenched to $0{ }^{\circ} \mathrm{C}$ in ca. $2 \mathrm{~min}$. Stored in ice, until the measurement, a small amount of wax crystal were loaded onto the glass slide inside a copper stage with a central window. During the measurement, the temperature of the copper stage was controlled at 0 ${ }^{\circ} \mathrm{C}$, using a circulating bath.

SANS. The small-angle neutron scattering (SANS) experiments were conducted at the small-angle neutron diffractometer KWS1 at the FRJ-2 research reactor in Jülich, Germany (see www.neutronscattering.de). The range of momentum transfer $(Q)$ was $0.002-0.2 \AA^{-1}$, which means that three measurements were made at different sample-to-detector distances (between 1 and $20 \mathrm{~m}$ ) and a neutron wavelength of $7 \AA$. The "raw" data from the samples were corrected from the scattering background and were determined in absolute units, using a secondary standard. The samples were positioned in an oven in a vacuum chamber in which the temperature was controlled between $-30{ }^{\circ} \mathrm{C}$ and $65{ }^{\circ} \mathrm{C}$ by a circulating bath.

\section{Results}

Yield Stress Rheological Measurements. Below the wax precipitation temperature, paraffin wax precipitates in platelike structures in decane. At sufficiently high wax concentrations, these platelets can form a

(18) Wardhaugh, L. T.; Boger, D. V. J. Rheol. 1991, 35, 1121. 


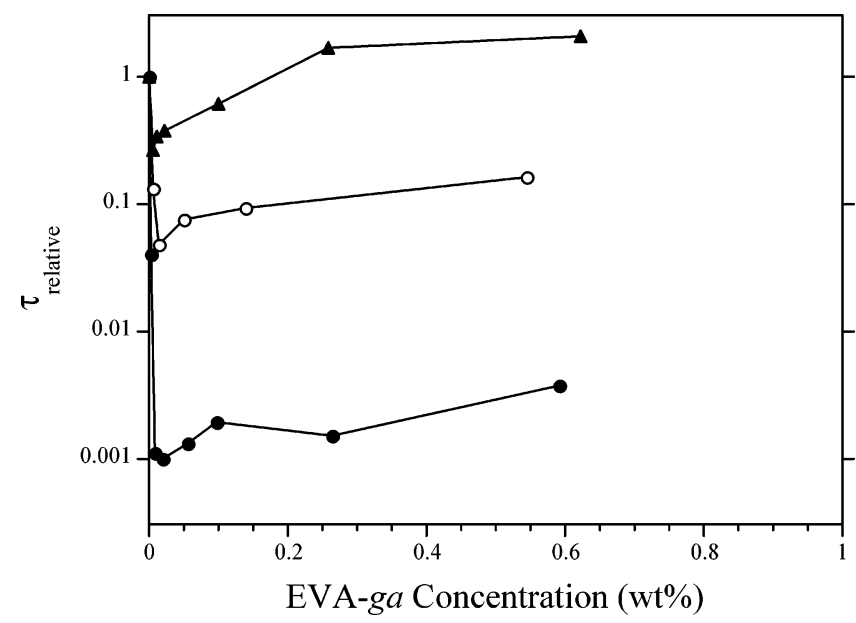

Figure 1. Effect of growth-arrestor-grade ethylene/vinyl acetate (EVA-ga) on the yield stress of $4 \mathrm{wt} \%$ paraffin wax gels in decane at $0{ }^{\circ} \mathrm{C}$, as a function of the wax modifier concentration. The relative yield stress is defined as the yield stress of the sample with modifier divided by the yield stress of the sample without modifier. The yield stresses of the unmodified wax gels is reported in ref 6 . Symbols correspond to results for $\mathrm{C}_{28}$ (filled triangles), $\mathrm{C}_{32}$ (open circles), and $\mathrm{C}_{36}$ (filled circles) waxes.

sample-spanning "house of cards" structure that entrains decane and forms a gel with a large yield stress. The 4 wt \% paraffin samples considered here readily form such gels. The objective of adding a wax modifier is to disrupt the formation of the gel. This occurs either by promoting the nucleation of multiple wax precipitation sites (and, thus, reducing the dimensions of the platelets) and/or adsorbing polymer brushes onto the platelets to prevent wax aggregation and collapse the house of cards. Diblock polymers such as polyethylenepoly(ethylene-propylene) (PE-PEP) utilize the latter mechanism, forming an amorphous PEP brush structure on the wax crystals, in addition to promoting wax nucleation. ${ }^{6}$ However, random co-polymers such as EVA most likely use the former mechanism, where the crystallizable methylene runs act as nucleation sites. This action is similar to what we previously observed for random co-polymers of PEB- $n .^{8-10}$ Moreover, we demonstrated that, depending on the degree of ethyl side branching of PEB- $n$, the selectivity of the polymer for disrupting the precipitation of paraffins of distinct chain lengths could be tuned, depending on the crystallization temperatures of the various segments. PEB- $n$ interactions with wax cease when $n>14,{ }^{19}$ which reflects the absence of methylene runs of sufficient lengths to crystallize.

A similar effect may be anticipated for EVA, depending on the polymer grade. The effect of EVA-ga and EVA-ss on the relative yield stresses of various 4 wt \% paraffin wax gels in decane at $0{ }^{\circ} \mathrm{C}$ is shown in Figures 1 and 2, respectively. EVA breaks down the gel structures of all the three paraffins considered. Indeed, additive concentrations of $\sim 100 \mathrm{ppm}(0.01 \mathrm{wt} \%)$ are sufficient to reduce the yield stress of the $\mathrm{C}_{36}$ gels by 3 orders of magnitude or more. Both additives show an increasing selectivity for breaking down gels formed by paraffins of increasing chain length. That is, the minimum in the yield stress obtained for the $\mathrm{C}_{36}$ wax is less

(19) Radulescu, A. Private communication.

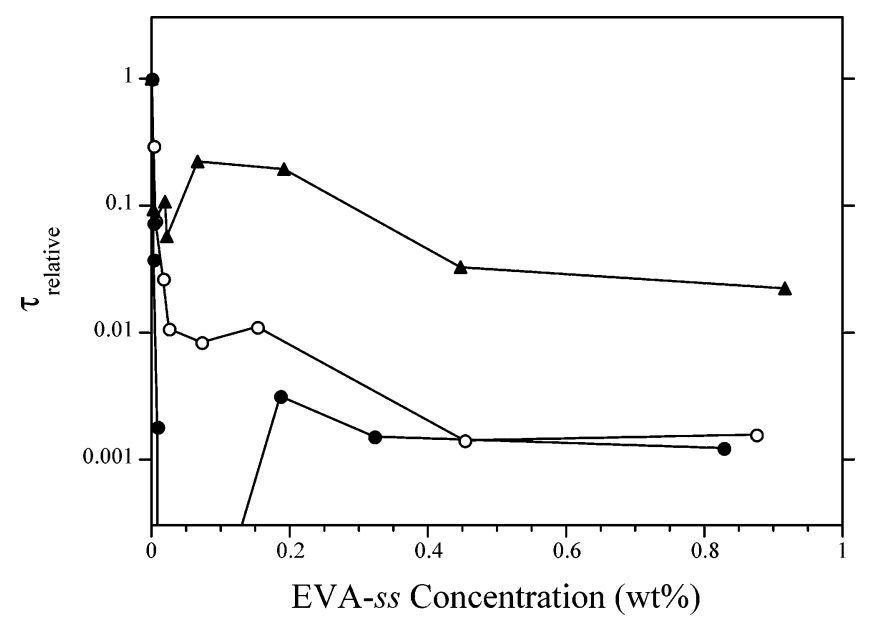

Figure 2. Effect of single-shot-grade ethylene/vinyl acetate (EVA-ss) on the yield stress of $4 \mathrm{wt} \%$ paraffin wax gels in decane at $0{ }^{\circ} \mathrm{C}$, as a function of the wax modifier concentration; the relative yield stress and symbols are as defined in the caption for Figure 1.

than that observed for the $\mathrm{C}_{32}$ wax, which, in turn, is lower than that observed for the $\mathrm{C}_{28}$ wax. Indeed, with increasing EVA-ga concentration, the yield stress of the $\mathrm{C}_{28}$ wax eventually becomes greater than that observed for the neat wax gel, i.e., the gel becomes stronger with increasing additive concentration (see Figure 1).

The two wax modifiers show interesting differences in their ability to break down the wax gels. The selectivity of EVA-ga is very pronounced, and there is good separation between the yield stress curves for the three paraffins considered (see Figure 1). In contrast, the EVA-ss grade seems to be most active at concentrations of $\sim 0.1$ wt $\%$, where there is a minimum in the relative yield stress for all the paraffins (see Figure 2). Indeed, for the $\mathrm{C}_{36}$ wax, the yield stress decreases to such low values at this EVA-ss concentration that the rheometer was unable to measure a yield stress. This low value (below the range of yield stress shown) simply reflects the minimum stress that the instrument can generate. At intermediate concentrations of $\sim 0.2 \mathrm{wt} \%$ EVA-ss, the yield stresses of the various paraffins pass through a maximum before decreasing again to plateau at higher EVA-ss concentrations. This plateau seems to be the same for the $\mathrm{C}_{32}$ and $\mathrm{C}_{36}$ waxes.

Optical Microscopy. Solutions of $4 \% \mathrm{C}_{36}$ in decane were chosen for investigation of the microscopic crystal morphology (Figures3 and 4). The pure $\mathrm{C}_{36}$ wax crystals exhibit a sheetlike structure (see Figure 4a) with sizes of $>100 \mu \mathrm{m}$, whereas $70 \mathrm{ppm}$ of EVA-ss reduces the size of the crystals to ca. $30 \mu \mathrm{m}$ (see Figure $4 \mathrm{~b}$ ). An average size of $<10 \mu \mathrm{m}$ occurs at a concentration of $0.097 \%$ EVAss, which coincides with the lowest yield stress in Figure 2 . Not only does the average particle size decrease, but the particle morphology also changes from platelike (see Figure 4a) to a more compact and spherical form (see Figures $4 \mathrm{c}-\mathrm{f}$ and $3 \mathrm{a}-\mathrm{d}$ ). The EVA-ga (contrary to its perceived commercial function as a growth arrestor) seems to have less impact on crystal size than does EVA-ss (see Figure 3b versus Figure $4 \mathrm{c}$ at $\sim 0.09$ wt \% EVA). This is consistent with the EVA-ss functioning as a nucleation agent as well as a growth arrestor.

Mechanistically, the notion exists that the activity of the EVA family is related to the polarity incumbent in 


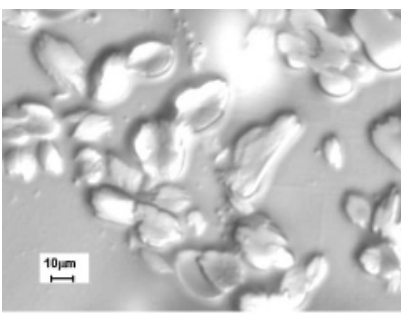

(a)

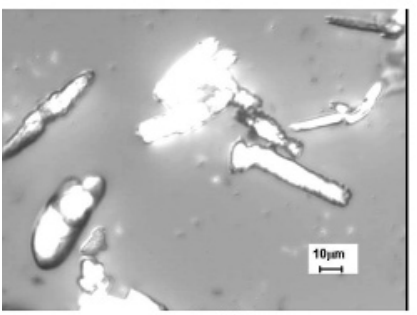

(c)

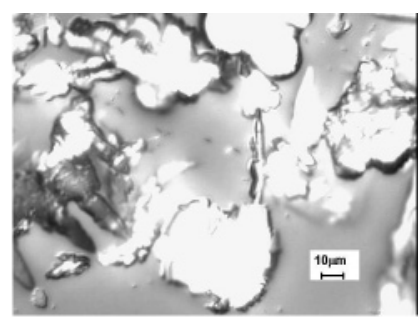

(b)

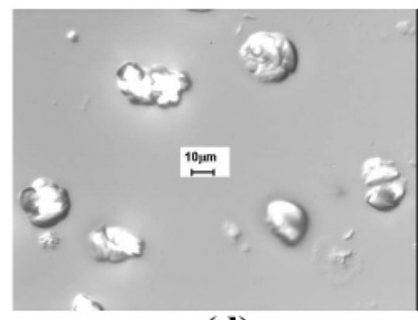

(d)
Figure 3. Optical micrographs of $4 \% \mathrm{C}_{36}$ with different amount of EVA-ga: (a) $0.008 \%$ EVA-ga, (b) $0.085 \%$ EVA-ga, (c) $0.255 \%$ EVA-ga, and (d) $0.600 \%$ EVA-ga.

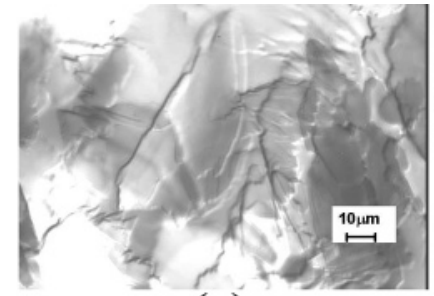

(a)

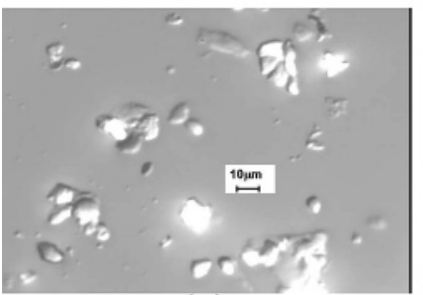

(c)

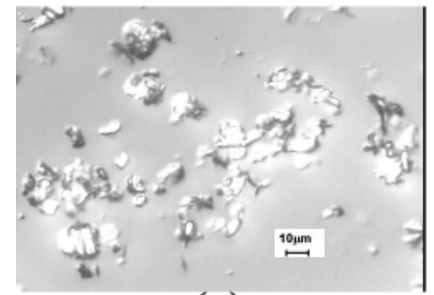

(e)

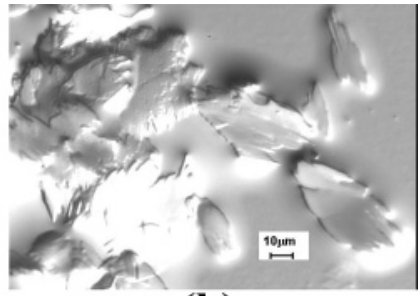

(b)

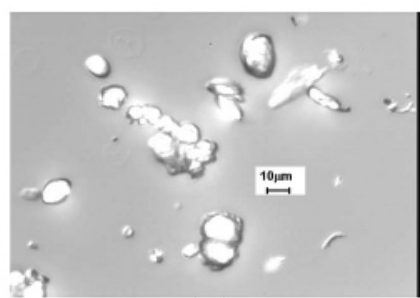

(d)

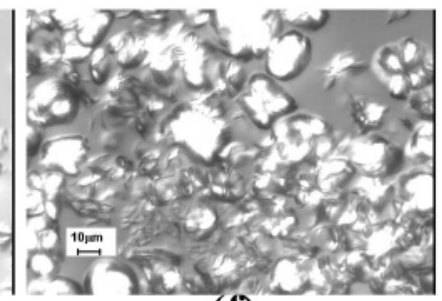

(f)
Figure 4. Optical micrographs of $4 \% \mathrm{C}_{36}$ with various amount of EVA-ss: (a) $4 \% \mathrm{C}_{36}$, (b) $0.007 \%$ EVA-ss, (c) $0.097 \%$ EVA-ss, (d) $0.185 \%$ EVA-ss, (e) $0.324 \%$ EVA-ss, and (f) $0.827 \%$ EVAss.

the VA content. ${ }^{20}$ However, the commercial PE-PEP diblocks and PEB- $n$ co-polymers contain no polar segments or units. The PE-PEP activity has been related ${ }^{5}$ to the calculated available plate surface area (APSA), with the measured activity expressible in terms of the cold filter plugging point (CFPP). The methodology by which the APSA is derived is given in ref 5 . When combined with the CFPP values, the emerging behavior

(20) Davidson, I. G.; Cameron, G. G. Macromolecules 1995, 28, 5964.
Table 1. Dependence of the Spatial Arrangement of the Polymers $(\alpha)$ on Aggregate Architecture

\begin{tabular}{ll}
\hline \multicolumn{1}{c}{$\alpha$} & \multicolumn{1}{c}{ architecture } \\
\hline 1 & rods \\
2 & plates \\
$<3$ & mass fractals \\
$>3$ and $<4$ & surface fractals \\
4 & 3-dimensional objects \\
$>4$ & diffuse interfaces
\end{tabular}

demonstrates that the surface area is jointly dependent on the PE-PEP segment molecular weights and their ratio. For example, a PE-PEP with $10 \mathrm{~K}-35 \mathrm{~K}$ segment molecular weights will yield a surface area of $\sim 115 \mathrm{~m}^{2} /$ $\mathrm{g}$, whereas the commercially viable $1.5 \mathrm{~K}-5 \mathrm{~K}$ PE-PEP sample yields a surface area of $\sim 250 \mathrm{~m}^{2} / \mathrm{g}$. Changing the $5 \mathrm{~K} \mathrm{PEP}$ segment to $11 \mathrm{~K}$ reduces the surface area to $\sim 210 \mathrm{~m}^{2} / \mathrm{g}$.

SANS. At high temperatures, when the polymers are completely dissolved in the solvent, the systems yield scattering characteristics that are typical of single coils. As the system temperature decreases, the self-assembly process results in the formation of large objects with a morphology that is characteristic to each of the systems. The scattering from these objects may be characterized by a power law: ${ }^{21}$

$$
I(Q)=\frac{\mathrm{d} \Sigma}{\mathrm{d} \Omega}(Q) \approx Q^{-\alpha}
$$

Here, $I$ denotes the intensity and the exponent $\alpha$ signifies the spatial arrangements of the polymers. The exponent $\alpha$ yields immediate semiquantitative insight into the morphology of the self-assembled aggregates (see below). In the case of single coils, $\alpha=1 / v$, where the Flory exponent assumes a value of $v=3 / 5$ for swollen chains and $v=1 / 2$ for Gaussian chains (the theta condition). The macroscopic cross section is

$$
\frac{\mathrm{d} \Sigma}{\mathrm{d} \Omega}(Q)=\Phi V_{\mathrm{w}}\left(\Delta \rho_{\mathrm{p}}^{2} / N_{\mathrm{A}}\right) P_{\mathrm{p}}(Q)
$$

Here, $V_{\mathrm{w}}$ is the polymer molar volume, $\Delta \rho_{\mathrm{p}}$ the difference between the scattering length densities of polymer and solvent, and $N_{\mathrm{A}}$ the Avogadro number.

Previous SANS measurements have shown that the PE-PEP diblock co-polymers form well-defined "hairy" plates $(\alpha=2),{ }^{5}$ whereas PEB- $n$ random co-polymers form needlelike structures $(\alpha=1){ }^{8-10}$ The interplay between the exponent $\alpha$ and the aggregate structure is summarized in Table $1 .^{21}$

In the presence of wax (e.g., $\mathrm{C}_{36}$ ), the crystallineamorphous random PEB- $n$ co-polymers form the hairy plate structure via co-crystallization with the wax. ${ }^{8-10}$ Thin plates form that are made of the wax. This in situ structure can then serve as a nucleating platform for the remaining wax in the system. The SANS scattering results for the EVA samples show that the self-assembled structures are more complicated than that observed for the low-molecular-weight PEB- $n$ co-polymers.

The scattering patterns from all EVA samples in $2 \%$ solutions are shown in Figures 5-7. We note the increase of scattering with decreasing temperature. The

(21) Higgins, J. S.; Benoit, H. Polymers and Neutron Scattering Clarendon Press: Oxford, England, 1994. 


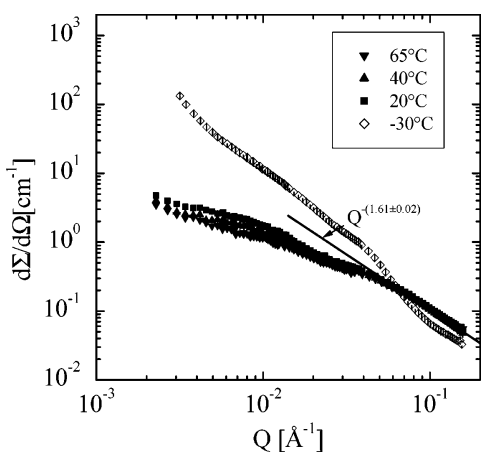

Figure 5. Scattering results of EVA-ga $2 \%$ solution at different temperatures.

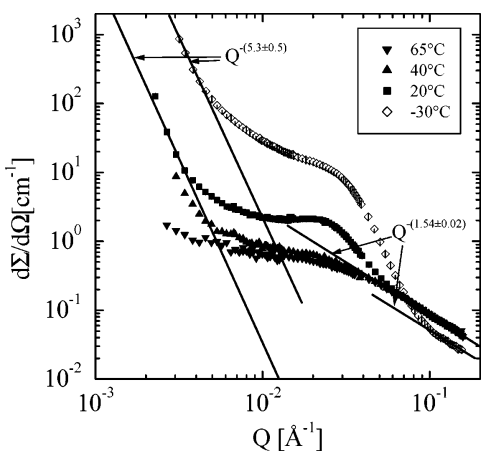

Figure 6. Scattering results of nucleator-grade ethylene/vinyl acetate (EVA-nu) $2 \%$ solution at different temperatures (data taken from ref 10).

observed temperature dependencies are significantly different for the different materials, with the nucleator showing the strongest scattering and the growth arrestor showing the weakest scattering, indicating different aggregation properties of the three polymers.

The scattering patterns from the EVA-ga are displayed in Figure 5. The intensity profiles for room temperature and above are almost identical, exhibiting a $Q^{-5 / 3}$ tail at high $Q$. At $-30{ }^{\circ} \mathrm{C}$, a strong intensity increase is observed, which exhibits a power law with an exponent of $\sim 1.6$, indicating a very open fractal aggregate of dimension $D=1.6$. At high $Q$, a significant decrease in intensity is observed, which most likely comes from the fact that a fraction of the EVA molecules precipitates from solution. ${ }^{9}$ This indicates that these commercial EVA materials contain an "inactive" fraction.

Figure 6 displays the temperature-dependent scattering results from the EVA-nu, which, having a lower VA content, contains longer methylene units with a high potential for crystallization and, therefore, aggregation. The crystallization event is favored after the mean sequence number of $\sim 20$ methylene units is achieved..$^{22}$ A distinct increase in the scattering already begins at temperatures of $\sim 40{ }^{\circ} \mathrm{C}$. The strong increase of scattering at low $Q$ is particularly noteworthy. Because only a narrow $Q$-range in the low- $Q$ regime is covered, determination of a power law is difficult. However, exponents of $>5$ are seemingly present. Such high exponents cannot be interpreted in terms of fractal aggregates or fractal surfaces and most likely originates from diffuse surface scattering, where the so-called Porod exponent 4 is further increased by a smeared-

(22) Davison, S.; Taylor, G. L. Br. Polym. J. 1972, 4, 65.

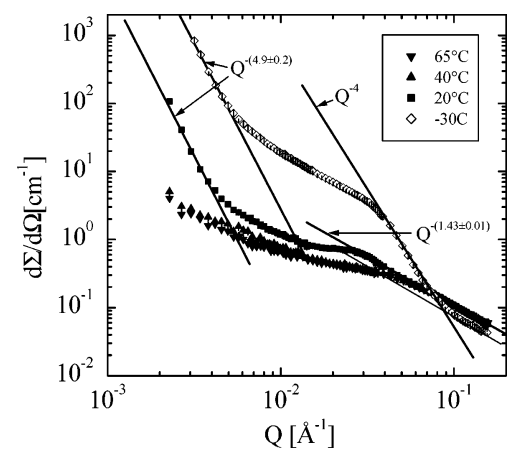

Figure 7. Scattering results of EVA-ss $2 \%$ solution at different temperatures.

out surface. If this interpretation is correct, then EVAnu forms large aggregates with pronounced surfaces and aggregate sizes well above several thousand angstroms. It is the existence of an aggregate surface that distinguishes the EVA-nu aggregates from those of the other materials. Therefore, EVA-nu must develop much more compact structures than the other aggregating polymers. This implies that the surface area available for wax nucleation is diminished. Based on these observations, the EVA-nu material has its potential activity masked by the formation of these complicated, largescale structures. Lucid control of the mean length of the methylene segments in the EVA-nu should enhance its wax-nucleating capacities. Such control could also eliminate partial precipitation of the EVA-nu, thus further improving its activity.

Another feature is the presence of a peak structure at $Q^{*} \approx 0.025 \AA^{-1}$. Such a peak indicates a structural correlation length of $R=2 \pi / Q \approx 250 \AA$. Finally, the loss of intensity at high $Q$ and low temperature could indicate either precipitation of part of the material or a loss of polymer scattering, because of an embedding of the chains into a more compact structure. Figure 7 displays the scattering pattern from the EVA-ss polymer at various temperatures. Because this polymer has a VA content that is intermediate between those of the other two EVA samples, we expect an aggregation and scattering behavior that is intermediate between those of the other two EVA samples.

Figure 7 displays the scattering pattern from the EVA-ss polymer at various temperatures. This polymer has a VA content that is intermediate between those of the other two EVA samples; therefore, we expect intermediate aggregation and scattering behavior. Although at $65^{\circ} \mathrm{C}$, the scattering patterns of the nucleator and the single-shot material are very similar, at $40{ }^{\circ} \mathrm{C}$, the growth arrestor already displays signs of significant aggregation, whereas the ss grade does not. Significant aggregation begins between $40{ }^{\circ} \mathrm{C}$ and $20{ }^{\circ} \mathrm{C}$. At room temperature and low $Q$, a very strong intensity increase is observed (gradient $\cong 4.9$ ), which is indicative of a fraction of large particles with sharp interfaces. At -30 ${ }^{\circ} \mathrm{C}$, the ss- and ga-grade polymers again exhibit rather similar patterns, with a generally lower intensity level for the single-shot system, indicating somewhat weaker aggregation. As for the growth arrestor, there again are signs for an intermediate length scale of $\sim 250 \AA$, the microscopic identification of which is still not clear. 


\section{Comparisons of EVA, PEB-n, and PE-PEP}

As room temperature is approached, the EVA solutions become cloudy and white sediment slowly accumulates at the bottom of the cell; this effect is enhanced at $-30^{\circ} \mathrm{C}$. This behavior is detrimental to the activity of the formulations, because of the removal of the self-assembled crystallized domains from solution. Although the large aggregates make the solution totally opaque to light, the transmission of neutrons is only slightly changed from 0.78 at room temperature to 0.73 at $-30{ }^{\circ} \mathrm{C}$. The scattering intensity at $-30{ }^{\circ} \mathrm{C}$ at $Q>$ $0.1 \AA^{-1}$ decreases by $23 \%$ in the EVA-ga sample, by $38 \%$ in the EVA-ss sample, and by $55 \%$ in the EVA-nu sample. Thus, the EVA samples are less soluble in decane and start to fractionate from solution at temperatures greater than the solubility limit of the PEB- $n$ additives. This is most pronounced for the EVA-nu grade. ${ }^{10}$ As a result, the fraction of the EVA polymers that are available to interact with the paraffin waxes is less certain than for the PEB- $n$ case.

In contrast, self-assembled aggregates of the prototype commercial diblock and the PEB-7.5 and PEB-11 random co-polymers remain in solution at $-30{ }^{\circ} \mathrm{C}$ and the scattering intensity at high $Q$ is independent of temperature. When $n$ reaches a value of $\sim 6$ or less for the PEB- $n$ series, precipitation from solution occurs. This is, of course, a consequence of an increase in the mean value of the methylene runs as the butene content is lessened.

The EVA-nu and EVA-ss materials display distinctly different aggregation properties, relative to EVA-ga. Although the former only weakly aggregates and seems to form very open fractal aggregates of a dimension $D$ $=1.6$, the other two materials form large-scale $(>3000-$ $4000 \AA$ in size) compact structures with a defined surface. This distinguishes them also from the random co-polymer PEB-11 (6K) materials, which form loose, network-like structures at low temperatures $\left(\sim 0{ }^{\circ} \mathrm{C}\right) .8$ The PEB-7.5 co-polymer (6K) commences to self-assemble at $\sim 50{ }^{\circ} \mathrm{C}$.

In particular, the lower the degree of ethyl side branching in the PEB- $n$ series, the greater the length of the constituent ethylene runs, which imparts a higher degree of selectivity for breaking down wax gels that are made from longer-chain paraffins. Here, we find similar selectivities of the EVA-ga and EVA-ss grades for the longest paraffin considered, despite the fact that EVA-ss has a lower degree of VA substitution (nominally, $12 \mathrm{~mol} \% \mathrm{VA}$ ), compared to EVA-ga (nominally, $15 \mathrm{~mol} \% \mathrm{VA}$ ). Moreover, at high additive concentrations, EVA-ss breaks down both $\mathrm{C}_{32}$ and $\mathrm{C}_{36}$ gels to a comparable extent, whereas EVA-ga displays a greater affinity for breaking down the gel structure of $\mathrm{C}_{36}$, compared to $\mathrm{C}_{32}$, over the entire concentration range. This counterintuitive behavior may be attributed in part to differences in the characteristics of the PEB- $n$ and EVA additives.

The co-polymerization of ethylene and VA can be classified $^{7}$ as almost ideal, because $r_{1} r_{2} \approx 1$ (where $r_{1}$ and $r_{2}$ are the reactivity ratios of ethylene and VA, respectively $\left(r_{1}=0.8\right.$ and $\left.r_{2}=1.2\right)$ ). Thus, monomer incorporation can be, to a first approximation, random in these EVA systems. Hence, higher VA concentrations should translate to shorter average mean sequence lengths of methylene segments. Thus, it is interesting to contemplate the self-assembly behavior of EVA materials with higher VA contents and smaller methylene runs. This could allow the EVA self-assembly event to form the orderly modulated needlelike micelles that are known to form ${ }^{8-10}$ from the crystalline/ amorphous PEB- $n$ random co-polymers, alternating multisegment atactic-iso-polypropylen $\mathrm{e}^{23}$ and starbranched co-polymers, ${ }^{10}$ where the crystalline fraction resides in the star interior. Thus, the formation of ethylene segments of limited length in a well-controlled free-radical polymerization could eliminate the precipitation event while simultaneously limiting (or eliminating) the formation of the large ill-defined self-assembled structures currently found for the EVA-no and EVAsis co-polymers.

Krishnamoorti determined ${ }^{24}$ the $r_{1} r_{2}$ products for 17 PEB- $n$ samples, where the value of $n$ ranged from $\sim 7$ to 45 . That $r_{1} r_{2}$ value was $0.73( \pm 0.06)$. This indicates a slight trend toward alternation in the PEB- $n$ series, whereas the corresponding value ${ }^{7}$ of $\sim 1$ for the EVA materials indicates a slightly more random mode for the incorporation of ethylene and VA than would be expected for the ethylene-butene system.

\section{Conclusions}

The ethylene/vinyl acetate (EVA) co-polymers reduce the yield stresses of $4 \mathrm{wt} \%$ wax solutions in decane. The reduction in yield stress is 3 orders of magnitude for the $\mathrm{C}_{36}$ wax; however, the reduction was 1 order of magnitude for the growth-arrestor-grade co-polymer (EVA-ga) with $\mathrm{C}_{32}$ and only 3 -fold for the $\mathrm{C}_{28}$ wax. The response of the yield stress to EVA addition is nonlinear and indicates saturation of the interaction between the polymer and wax. Increasing the dosage level above a few hundred parts per million has no further effect on reducing the yield stress. Especially for the $\mathrm{C}_{28}$ wax, neither the EVA-ga nor the single-shot-grade co-polymer (EVA-ss) would provide an adequate reduction in yield stress to ensure against gelation in pipeline transport.

Micrographs of the wax crystal morphology indicate that the EVA-ga co-polymer changes the wax crystal habit at higher concentrations more effectively than does the EVA-ss, which is identified as a combination of nucleator (EVA-nu) and growth arrestor (EVA-ga). The EVA-ss seems to form more wax crystals at low concentrations than does the EVA-ga, which would be consistent with its role as a nucleating agent. However, it is clear that the commercial designations as "growth arrestor" and "nucleator" are heuristic and do not explain mechanistically the role of these polymers in modifying wax crystal structure or wax crystal interactions. Further molecular level work must be conducted to fully clarify the mechanisms involved.

Neutron scattering studies of the self-assembly of the EVA polymers, as a function of temperature, show dramatically different aggregated structures in decane. At higher temperatures $\left(20,40\right.$, and $\left.60{ }^{\circ} \mathrm{C}\right)$, the EVAga with the lowest level of crystallinity shows that the scattering intensity increases as $Q$ increases at low $Q$

(23) Radulescu, A.; Mathers, R. T.; Coates, G. C.; Richter, D.; Fetters, L. J. Macromolecules 2004, 37, 6962. 49.

(24) Krishnamoorti, R. Ph.D. Thesis, Princeton University, 1994, p 
values. This indicates partial aggregation that is similar at all temperatures. In contrast, EVA-ss, with higher levels of crystallinity, shows aggregation that decreases as the temperature increases. However, both of these behaviors are different from our previous studies on lower-molecular-weight polyethylene-poly(ethylenepropylene) (PE-PEP) block co-polymers and poly(ethylene-butylene) (PEB- $n$ ) co-polymers with controlled microcrystallinity. For both of these polymers, at $60{ }^{\circ} \mathrm{C}$, the polymers were completely dissolved and single-chain scattering was observed. ${ }^{8-10}$ At low temperature $\left(-30^{\circ} \mathrm{C}\right)$, the EVA-ga shows scattering that continuously increases, with a power law exponent of 1.6. This behavior is observed for weakly aggregating polymer gels. In contrast, the EVA-ss at 40, 20, and -30 ${ }^{\circ} \mathrm{C}$ shows a transition from surface scattering on strongly segregated objects for $Q<0.003 \mathrm{~A}^{-1}$ to a plateau whose height is dependent on temperature, to scattering with the power-law scaling of a chain in a good solvent for $Q$ $>0.3 \mathrm{~A}^{-1}$. A full investigation using the power of neutron scattering would require the synthesis of deuterated EVA samples. This would parallel the work that we have previously presented on PEB samples that are made via anionic synthesis. At this point, the synthesis of the corresponding EVA samples is not feasible.
In this study, we have not investigated the changes in rheology at the different temperatures where we have studied scattering. The difference in aggregation versus temperature may correlate with the sensitivity of the yield stresses to wax molecular weight (at $0{ }^{\circ} \mathrm{C}$ ). We have studied these effects for PEB- $n$ and PE-PEP and have found that the most effective gelation inhibitors are those that aggregate at the temperature of the wax crystallization event.

In addition to these studies of wax gelation and polymer self-assembly, another important question is the deposition of waxes on surfaces. The inhibition of wax deposition by the polymers is currently being investigated.

Acknowledgment. We would like to acknowledge financial support from Halliburton Energy Services Company. We appreciate the comments by one of the reviewers, summarizing the current understanding in the field and the need for three-dimensional imaging and crystal growth mechanism studies.

EF049910I 